\title{
The Fate of Cosmic String Zero Modes
}

\author{
A. C. DAVIS \\ Department of Applied Mathematics and Theoretical Physics, \\ Centre for Mathematical Sciences, \\ University of Cambridge, Cambridge, CB3 OWA, UK \\ S. C. DAVIS and W. B. PERKINS \\ Department of Physics, University of Wales Swansea, \\ Singleton Park, Swansea, SA2 8PP, UK
}

\begin{abstract}
Cosmic strings in realistic particle physics models have fermion zero modes in the string core. Such zero modes change the underlying cosmology; for example, the string can carry a current. We consider the stability of fermion zero modes to interactions with particles in the surrounding plasma. We also consider cosmological constraints on such theories, particulary those with a chiral fermion zero mode, such as arises in a class of supersymmetric models.
\end{abstract}

\section{Introduction}

Although much of the evolution of the Universe is well understood, there are still many cosmological phenomena for which a completely satisfactory explanation has yet to be found. Topological defects, such as cosmic strings, could provide mechanisms for structure formation, CMB anisotropy, and high energy cosmic rays 1 . Such defects form in many realistic particle physics theories, including those involving supersymmetry.

In the past it has been difficult to evaluate the usefulness of such ideas due to a lack of data. This is now changing, and predictions of CMB anisotropies from simple cosmic string models have been madel. While these predictions show poor agreement with the observations, they do not take into account the full physics of string models. Indeed, recent analysis which includes the effect of particle production as an energy loss mechanism from the string network shows much improved agreement with data 3 .

One significant possibility is that the strings carry conserved currents 1 . These currents will alter the evolution of a string network, which could lead to better agreement with observation. Indeed, an analytic analysis showed that a much denser string network results for electromagnetically coupled strings $\mathrm{E}$. However, one significant implication of conserved currents is that they can stabilise loops of string. If persistent, these stabilised loops or 'vortons' can easily dominate the energy density of the Universe, placing stringent constraints on the parameters of the model. An analysis has been made of the 
implications of this for particle physics models that predict current carrying strings $\mathrm{B}$.

Fermions are a natural choice for the charge carriers of such currents. Fermion zero modes exist in a wide class of cosmic string models. The fermions can be excited and move along the string, resulting in a current. Cgnsequently, fermion conductivity occurs naturally in many supersymmetric 10 and grand unified theories 11 , such as $\mathrm{SO}(10)$. In particular, in SUSY models with a D term, there is a single chiral zero mode, either a left or right mover. This zero mode survives supersymmetry breaking 12 . This class of theory is potentially important since it arises in many superstring models.

One criticism of fermion currents is that, unlike scalar boson currents, they are not topologically stable. It is possible that they could decay, either directly into particles off the string, or through interactions with the surrounding plasma. If the decay rate is too high, currents will not last long enough to have any significant effect. On the other hand if the decay rate is too low the Universe could become vorton dominated. We address this issue and show that these processes can remove current carriers close to the phase transition, but not otherwise 13. We then consider vorton constraints in chiral theories, showing this leads to stringent constraints on the underlying particle physics theory.

\section{$2 \quad D$-term Supersymmetric Cosmic Strings}

Consider a supersymmetric theory with a $\mathrm{U}(1) \rightarrow I$ phase transition. The simplest way to achieve this symmetry breaking is to use one charged chiral superfield and a non-zero Fayet-Iliopoulos term. Expanding the Lagrangian in terms of component fields gives (in Wess-Zumino gauge)

$$
\begin{array}{r}
\mathcal{L}=\mid\left(\left.D_{\mu} \phi\right|^{2}-\frac{1}{4} F^{\mu \nu} F_{\mu \nu}+|F|^{2}+\frac{1}{2} D^{2}+D\left(g|\phi|^{2}+\xi\right)\right. \\
-i \psi \sigma^{\mu} D_{\mu}^{*} \bar{\psi}-i \lambda \sigma^{\mu} \partial_{\mu} \bar{\lambda}+i g \sqrt{2} \phi^{*} \psi \lambda+\text { (c.c.) } .
\end{array}
$$

The two fermion fields $\psi$ and $\lambda$ are the Higgsino and gaugino respectively. Eliminating the two auxillary fields $D$ and $F$ will give potential terms. If $\xi=-g \eta^{2}<0$ the resulting $D$-term will give rise to spontaneous symmetry breaking, resulting in an expectation value of $\eta$ for $\phi$. In order to avoid gauge anomalies, the model must contain other charged superfields. We will assume these have zero expectation values.

As well as the $\phi=$ constant solution there also exist string solutions obtained from the ansatz

$$
\phi=\eta e^{i n \theta} f(r)
$$




$$
\begin{aligned}
A_{\mu} & =-n \frac{a(r)}{g r} \delta_{\mu}^{\theta} \\
D & =g \eta^{2}\left(1-f(r)^{2}\right),
\end{aligned}
$$

where $f(r)$ and $a(r)$ are the usual Neilsen-Olesen profile functions.

Now consider the fermionic sector of the theory. Performing a SUSY transformation with 2-spinor parameter $\epsilon$ gives

$$
\begin{aligned}
& \delta \lambda_{1}=2 i g \eta^{2}\left(1-f^{2}\right) \epsilon_{1} \\
& \delta \psi_{1}=-2 \sqrt{2} i \eta \frac{n}{r}(1-a) f e^{i(n-1) \theta} \epsilon_{1}^{*} .
\end{aligned}
$$

$\delta \psi_{2}$ and $\delta \lambda_{2}$ are both zero. The expression (5) is a zero energy solution of the fermion field equations. It is trivial to add $z$ and $t$ dependence to it, and the resulting solution is a null current moving along the string.

Since the string solution is not invariant under transformations with $\epsilon_{1} \neq 0$, supersymmetry has been broken inside the string core. However it is only partially broken there since the string is still invaraint under transformations parametrised by $\epsilon_{2}$.

It is also possible to get a phase transition using an $F$-term. This requires a non-trivial superpotential and at least three scalar fields. The corresponding string solutions have twice as many zero modes as the $D$-term theory, and move in both directions along the string. In this case supersymmetry is completely broken 1 . In both theories the zero modes and SUSY breaking are confined to the string core.

When soft supersymmetry-breaking terms are included in the Lagrangian, oppositely moving zero modes in the $F$-term theory mix to form massive states 12 . This cannot happen in the $D$-term theory since the zero modes all move in the same direction.

Thus, a generic property of cosmic strings in SUSY theories is that supersymmetry is broken in the string core and the resulting strings have fermion zero modes. As a consequence, cosmic strings arising in SUSY theories are automatically current-carrying and can give rise to vortons.

\section{Decay Rates of Charge Carriers}

Vortons can only be stable if the currents they carry are stable, thus the stabilty of the charge carriers is a crucial consideration. In the absence of other particles, currents carried by zero modes on isolated, straight strings are stable on grounds of energy and momentum conservation. However, in a realistic setting there are many processes which can depopulate zero modes. 
Strings are not isolated and in the early Universe they and their bound states will interact with the hot plasma. $\mathrm{SO}(10)$ is considered as a specific example. In this case a heavy neutrino zero mode may scatter from a light plasma particle to produce a light fermion-antifermion pair via an intermediate electroweak Higgs boson. A massive neutrino bound mode may also simply decay into light fermions. Further, bound states on different strings, or different parts of a single curved string, can scatter from one another by exchanging a Higgs particle.

The decay rates of both massive and massless modes have been calculated at tree level13. The main difference between scattering in the string background and that in a trivial background is the lack of translation invariance transverse to the string. This enters the calculation via the bound mode wavefunctions which are localised around the string. Integration over the initial vertex position does not yield the standard momentum conserving $\delta$-function, instead it produces an approximately gaussian function that permits non-conservation of transverse momentum on the scale of the off-string fermion mass.

The violation of momentum conservation in the string background opens up significant areas of phase space that are forbidden in the trivial background. Of particular importance is the possibility of resonant scattering. For example the lifetime of a bound mode of mass $m_{\mathrm{B}}$ decaying into a light fermion and an electroweak Higgs is,

$$
\tau \sim\left(\left|g_{\nu}\right|^{2} m_{\mathrm{B}}\right)^{-1},
$$

where $g_{\nu}$ is the Yukawa coupling in the neutrino's electroweak mass term. Massive modes with the appropriate couplings to the electroweak sector thus have a very short lifetime. The above result neglects the possibility that incoming and outgoing fermionic wavefunctions may be amplified near the string 14 and should be taken as an overestimate.

The fate of massless bound states is also complicated by momentum nonconservation. Of particular interest are the bound states that stabilise vortons. Contraction of the vorton will ensure that the states at the Fermi surface will have GUT scale momenta. The lifetime of these high momentum states is critical; if they decay the vorton will contract and promote low momentum states to high momentum. Energy and $z$-momentum conservation prevent massless states from decaying spontaneously. However it is possible for them to decay by interaction with plasma particles or other zero modes. If the string Higgs mass, $m_{\mathrm{s}}$ exceeds the off-string fermion mass, $m$, it is also possible for high energy massless currents on a curved string to decay by tunneling to free heavy neutrinos 6 . However the rate will not be significant unless $m_{\mathrm{s}} \gg m$.

If we consider a massless bound state at the Fermi surface scattering from a typical plasma particle, we have a centre of mass energy of order $\sqrt{m_{\mathrm{s}} T}$. 
In the $\mathrm{SO}(10)$ case this is well above the mass of the electroweak Higgs intermediate particle and transverse momentum non-conservation again allows for resonant scattering. Including amplification of the incoming plasma particle wavefunction, the lifetime of these high momentum zero modes is found to be,

$$
\tau \sim \frac{\widetilde{m}^{2}}{\left|g_{\nu}\right|^{2} T^{3}}\left(\frac{T}{\widetilde{m}}\right)^{2 Q}
$$

where $\widetilde{m}=m_{\mathrm{s}} m / 2$ and $Q$ is the charge of the plasma particle under the string gauge field.

In the radiation dominated era the time is given by $t=\alpha T^{-2}$, where $\alpha \sim m_{\mathrm{Pl}} / 10$. In the case of $\mathrm{SO}(10), Q=3 / 10$, and the probability of a zero mode state scattering after some time $t_{i}$ is small if,

$$
t_{i}>O\left(\left[\widetilde{m}^{-7}\left|g_{\nu}\right|^{10} \alpha^{6}\right]\right)>O\left(\left[\frac{m_{\mathrm{P} 1}}{10 \widetilde{m}}\right]^{6}\left|g_{\nu}\right|^{10} \widetilde{m}^{-1}\right) .
$$

As the lifetime varies only slightly faster than $T^{-2}$, this result for $t_{i}$ is very sensitive to the Yukawa coupling. For $\widetilde{m} \sim 10^{15} \mathrm{GeV}$, if $\left|g_{\nu}\right|=1$, zero mode states populated after $t_{i} \sim 10^{15} t_{\mathrm{GUT}}$ will be stable, while if $\left|g_{\nu}\right|<0.03$, this scattering is never significant. In the $\mathrm{SO}(10)$ model $g_{\nu}$ is also the Yukawa coupling for the corresponding quarks, thus there is an epoch when $\nu_{\tau}^{c}$ zero modes will scatter from the string, but $\nu_{e}^{c}$ and $\nu_{\mu}^{c}$ zero modes will never scatter by this process. Thus the interaction with plasma particles can not significantly remove zero modes from the string. Note that it is also possible to create currents using the above interactions in reverse. Hence, if thermal equilibrium is reached the number density of zero modes will be of order $T$.

Within the $\mathrm{SO}(10)$ model there is also the possibility of mediating these processes by GUT mass Higgs fields with zero VEV. In this case the Yukawa coupling need not be small, but the centre of mass energy of the interaction is only of order the intermediate particle mass for $T \sim T_{\mathrm{GUT}}$. Thus below the GUT temperature the reaction rates for these processes are rapidly suppressed by powers of $T / T_{\mathrm{GUT}}$.

None of these plasma scattering processes can remove $\nu_{e}^{c}$ and $\nu_{\mu}^{c}$ zero modes, and are only significant for $\nu_{\tau}^{c}$ immediately after the phase transition. Thus, they are unable to prevent the vorton density from dominating the energy density of the Universe.

The plasma scattering processes considered above failed to remove zero modes due to the decreasing plasma density at late times. A distinct category of process is the scattering of a zero mode on one string by a second zero mode on another string. This is particularly relevant for vortons as they form small 
loops with a typical radius only one or two orders of magnitude larger than the string width. We thus have zero modes moving in opposite directions on opposite sides of the vorton. For simplicity the decay rate can be calculated by considering two straight, anti-parallel strings 13 with spacing $2 R$.

If the intermediate particle is an electroweak Higgs boson, $R$ is much smaller than the electroweak length scale and there is no exponential range suppression. Resonant scattering is not possible and the cross-section is found to be,

$$
\sigma \sim \frac{\left|g_{\nu}\right|^{4}}{\left(m_{\mathrm{GUT}} R\right)^{4}} .
$$

This cross-section is dimensionless as the scattering is effectively in one spatial dimension.

Conversely, for a GUT mass intermediate particle, $m_{\mathrm{GUT}} R \sim 10-100$ and the reaction rate displays exponential range suppression,

$$
\sigma \sim \frac{\left|g_{\nu}\right|^{4}}{\left(m_{\mathrm{GUT}} R\right)^{3}} e^{-4 m_{\mathrm{GUT}} R} .
$$

While the string density may be high at formation, it drops rapidly and the exponential suppression in (10) makes such processes irrelevant in all physical situations.

Taken at face value, if electroweak particles mediate current-current scattering on different segments of string then (9) gives a short lifetime for charge carriers on a vorton. However, for a circular loop the angular momentum of the fermions must be conserved and, combined with energy conservation, this would prevent massless modes on the string scattering into massive modes. However, the fermionic spectrum has not been calculated for a circular loop, so there is no reason to expect the zero modes to remain massless. Thus angular momentum conservation can only be considered in a consistent, rotationally invariant calculation. The calculation above works consistently with straight strings, thus while (9) may not be directly applicable to vorton decay, it is relevant for interactions on non-circular loops and scattering of currents on a string network.

\section{Chiral Strings and Vortons}

We have seen that plasma interactions donot significantly remove zero modes from the string. This is particularly true in SUSY D-term theories (see section 21) where we would expect the zero mode to be isolated from the electroweak sector. As a consequence, stable vortons would be expected to form. In this section we constrain the underlying particle physics theory which gives 
rise to chiral zero modes on the string. We assume that the strings are formed at a phase transition ocurring at temperature $T_{\mathrm{x}}$ and become current-carrying at a scale $T_{\mathrm{s}}$. The string loop is characterised by two currents, the topologically conserved phase current $N$ and the dynamically conserved particle number current $Z$. In the chiral case these are exactly identical.

A non conducting string loop must ultimately decay by radiative and frictional drag processes until it disappears completely. However, a conducting string loop could reach a state in which the energy attains a minimum for given non zero values of $N$ and $Z$. This is the vorton state. It should be emphasised that the existence of such vorton states does not require that the carrier field be electromagnetically coupled. Indeed, in the case of the $D$-term zero modes, it is not.

The physical properties of a vorton state are determined by the quantum numbers, $N$ and $Z$. However, these are not arbitrary. Indeed, to avoid the fate of the usual loops, the quantum numbers on a conducting loop must be large compared with unity. This in turn implies a minimum length for the loop. In our work we calculate the number density of protovorton loops, subject to the loops being greater than a minimum length. We then estimate the vorton density and constrain the underlying theory by the requirement that the universe is not vorton dominated. We apply two constraints. Firstly we take a conservative assumption that the vortons only live a few minutes and constrain the theory by requiring that the universe is radiation dominated at nucleosynthesis; we then take the more realistic assumptions that chiral vorton loops are stable and constrain the theory by requiring that the vorton density is less than the closure density today. The full details are presented in ref. 6 ; here we sumarise the main results.

For chiral vortons the vorton energy, $E_{\mathrm{v}}$, is given by

$$
E_{\mathrm{v}} \simeq N T_{\mathrm{x}} .
$$

In the friction dominated era the number density of vortons can be estimated by considering the damping length scale and the resulting correlation length below which microstructure is damped. This automatically satisfies the minimum length criteria mentioned above. For a loop of length L, the conserved quantum number is then

$$
|Z|=N \approx L T_{\mathrm{s}}=\left(\frac{m_{\mathrm{P} 1}}{T_{\mathrm{s}}}\right)^{1 / 2} \frac{T_{\mathrm{x}}}{T_{\mathrm{s}}} .
$$

The resulting vorton density was found to be

$$
\frac{\rho_{\mathrm{v}}}{T^{3}} \approx \frac{T_{\mathrm{s}}^{3}}{m_{\mathrm{P} 1} T_{\mathrm{x}}},
$$


where factors of order unity have been dropped.

Requiring then that this vorton density be less than the radiation density at nucleosynthesis gives a constraint for strings which become current-carrying at formation of

$$
T_{\mathrm{x}} \leq 10^{8} \mathrm{GeV} .
$$

This is the condition that must be satisfied by the formation temperature of cosmic strings that become current-carrying immediately, subject to the rather conservative assumption that the resulting vortons last for at least a few minutes.

If the zero modes condense on the string at a separate phase transition then the constraint takes us outside the friction dominated regime. The calculation is much more involved and we refer the reader to ref. $\mathrm{g}$ for details. However, the resulting constraint is that GUT scale strings becoming current-carrying at a temperature above $10^{9} \mathrm{GeV}$ are inconsistent with data.

Being less conservative we can make the assumption that the vortons are absolutely stable and survive to the present time. Requiring that the vorton density is less than the closure density gives a stronger constraint. In this case we find

$$
T_{\mathrm{x}}=T_{\mathrm{s}} \leq 10^{5} \mathrm{GeV} .
$$

For strings which become current-carrying at a later transition, the details are again more complicated. However, for GUT scale strings we find that strings becoming current-carrying at a temperature above $10^{5} \mathrm{GeV}$ are inconsistent with data.

It is amusing to point out that if strings formed, or became currentcarrying, just below this temperature, then their vortons would contribute substantially to the dark matter of the universe.

\section{Discussion}

We have shown that cosmic strings arising in SUSY theories are generically current-carrying. For D-term theories there is a chiral fermion zero mode which survives SUSY breaking. Consequently, the current persists.

We have investigated ways in which the fermion bound modes could be destabilised. Zero modes are the most resilient: various scattering mechanisms were investigated, but, because of the low density of the surrounding plasma, they were unable to scatter all zero modes off the string. Zero modes on neighbouring string segments can also scatter off each other. This may be relevant for vortons.

Strings with a chiral zero mode lead to the most stringent constraints on the underlying particle physics theory, because in this case the fermion 
travels in a single direction, resulting in the current being maximal rather than random. We have shown that this leads to very strong constraints indeed.

However, if the theory somehow manages to evade the vorton constraints then the resulting cosmology could be very different. For example, the scaling solution of this type of cosmic string theory is completely unknown. Whilst the cosmology of strings resulting from the abelian Higgs model has been investigated, that resulting from realistic GUT theories has not. As a consequence. it is premature to rule out cosmic strings as being incompatible with microwave background data. This is currently under investigation 15 .

\section{Acknowledgments}

This work was supported in part by PPARC and an ESF network. We wish to thank our collaborators Brandon Carter and Mark Trodden, and also Rachel Jeannerot and the other organisers of COSMO99.

\section{References}

1. P. Bhattacharjee, Phys. Rev. D 40, 3968 (1989); E. M. Chudnovsky, G. B. Field, D. N. Spergel and A. Vilenkin, Phys. Rev. D 34, 944 (1986).

2. N. Turok, U. L. Pen and U. Seljak, Phys. Rev. D 58, 023506 (1998).

3. C. Contaldi, M. Hindmarsh and J. Magueijo, Phys. Rev. Lett. 82, 679 (1999); A. Riazuelo, N. Deruelle and P. Peter, astro-ph/9910290

4. E. Witten, Nucl. Phys. B 249, 557 (1985); R. Jackiw and P. Rossi, Nucl. Phys. B 190, 681 (1981).

5. K. Dimopoulos and A.C. Davis, Phys. Rev. D 57, 692 (1998).

6. R. L. Davis, Phys. Rev. D 38, 3722 (1988).

7. R. L. Davis and E. P. S. Shellard, Nucl. Phys. B 323, 209 (1989).

8. R. Brandenberger, B. Carter, A.C. Davis and M. Trodden, Phys. Rev. D 54, 6059 (1996).

9. B. Carter and A.C. Davis, Phys. Rev. D 61, 123501 (2000).

10. S.C. Davis, A.C. Davis and M. Trodden, Phys. Lett. B 405, 257 (1998).

11. A. Stern and U. A. Yajnik, Nucl. Phys. B 267, 158 (1986); S.C. Davis and A.C. Davis, Phys. Rev. D 55, 1879 (1997).

12. S.C. Davis, A.C. Davis and M. Trodden, Phys. Rev. D 57, 5184 (1998).

13. S.C. Davis, W.B. Perkins and A.C. Davis, hep-ph/9912356, Phys. Rev. $\mathrm{D}$, (in press).

14. W. B. Perkins, L. Perivolaropoulos, A. C. Davis, R. H. Brandenberger and A. Matheson, Nucl. Phys. B 353, 237 (1991).

15. A.C. Davis, T.W.B. Kibble, M. Pickles and D.A. Steer DAMTP-2000-46. 